\title{
Head and neck posture in sauropod dinosaurs inferred from extant animals
}

Michael P. Taylor, Mathew J. Wedel, and Darren Naish

Acta Palaeontologica Polonica 54 (2), 2009: 213-220 doi:http://dx.doi.org/10.4202/app.2009.0007

The neck posture of sauropod dinosaurs has long been controversial. Recent reconstructions position the cervical vertebrae and skull in an "osteological neutral pose" (ONP), the best fit arrived at by articulating the vertebrae with the zygapophyses in maximum contact. This approach in isolation suggests that most or all sauropods held their necks horizontally. However, a substantial literature on extant amniotes (mammals, turtles, squamates, crocodilians and birds) shows that living animals do not habitually maintain their necks in ONP. Instead, the neck is maximally extended and the head is maximally flexed, so that the mid-cervical region is near vertical. Unless sauropods behaved differently from all extant amniote groups, they must have habitually held their necks extended and their heads flexed. The life orientation of the heads of sauropods has been inferred from the inclination of the semi-circular canals. However, extant animals show wide variation in inclination of the "horizontal" semi-circular canal: the orientation of this structure is not tightly constrained and can give only a general idea of the life posture of extinct animals' heads.

Key words: Dinosauria, Sauropoda, extant amniotes, posture, neck, head, semi-circular canals.

Michael P. Taylor [dino@miketaylor.org.uk] and Darren Naish [eotyrannus@gmail.com ], Palaeobiology Research Group, School of Earth and Environmental Sciences, University of Portsmouth, Burnaby Building, Burnaby Road, Portsmouth PO1 3QL, UK; Mathew J. Wedel [mathew.wedel@gmail.com], Department of Anatomy and College of Podiatric Medicine, Western University of Health Sciences, 309 E. Second Street, Pomona, California 91766-1854, USA.

This is an open-access article distributed under the terms of the Creative Commons Attribution License (for details please see creativecommons.org), which permits unrestricted use, distribution, and reproduction in any medium, provided the original author and source are credited. 
For 5 Full text (404.1 kB) 